\title{
AN EVALUATION OF THE INTEGRATION OF STANDARDS AND GUIDELINES IN COMMUNITY PHARMACY PRACTICES.
}

\section{Authors}

$\mathrm{H}_{\text {Laetitia Hattingh }}{ }^{1}$

Michelle A King ${ }^{1}$

Nerida A Smith ${ }^{1}$

No of words in article

3,836

No of words in abstract

281

No of words in reference section

705

Affiliation of each author

1 School of Pharmacy, Griffith Health, Gold Coast campus, Griffith University Address for correspondence

Laetitia Hattingh

School of Pharmacy, Gold Coast campus, Griffith University, Queensland 4222

Email: I.hattingh@griffith.edu.au

Ph: $\quad+61755527097$

Fax: +61755528804 


\section{ABSTRACT}

\section{Objective}

To explore the usefulness and implementation of practice standards in community pharmacy practice regarding contemporary core services.

\section{Setting}

Community pharmacies in South East Queensland, Australia.

\section{Method}

During August and September 2006 semi-structured interviews with community pharmacists explored practice processes and procedures and the utilisation of endorsed standards and guidelines. Thematic content analysis of the interviews, inductive analysis and continual comparison of categories and concepts enabled common and distinct themes to be clarified.

\section{Main outcome measure}

Usefulness and integration of practice standards in the provision of core pharmacy services, utilisation of support staff, record keeping and overall risk management processes in community pharmacy.

\section{Results}

Seventeen community pharmacists participated, representing a wide range of demographics, experience and pharmacy types. Staff utilisation and record keeping practices were analysed as well as two core services, namely: (1) the supply of over-the-counter medicines, and (2) the dispensing of prescriptions. 
The procedures followed concerning the supply of over-the-counter medicines varied. The majority of participants did not regard this as a pharmacist's immediate role, but rather relied on support staff to identify when the supply required a pharmacist's intervention. Whilst all participants involved dispensary assistants in the dispensing process, the delegation of procedures differed. Most participants did not clearly differentiate between pharmacists' and dispensary assistants' activities. Dispensing processes and the involvement of pharmacists in the provision of patient advice varied. Pharmacist intervention record keeping was uncommon and records of 'near misses' were not routinely kept by any of the participants.

\section{Conclusion}

A lack of integration of practice standards indicated a need to review the standards for relevance. Additionally, pharmacists need to re-evaluate workflow models and the delegation of tasks in the light of new roles and responsibilities.

\section{KEY WORDS}

Practice standards - Quality healthcare - Risk management - Interviews Pharmacy - Australia - Pharmaceutical services - Pharmacy administration Professional practice

\section{IMPACT OF THE FINDINGS ON PRACTICE}

- The integration of prescribed standards differed amongst participants.

- The usefulness of practice standards should be re-evaluated. 
- The procedures for supply of over-the-counter medicine varied between pharmacies; supply was not regarded as a pharmacist's direct role.

- Workflow models and the delegation of tasks need to be re-evaluated in the light of new roles and responsibilities.

- Recording of pharmacist interventions is relatively poor and is not supported by dispensing software programs. 


\section{INTRODUCTION}

The changes in pharmacy practice in Australia are placing a greater emphasis on the provision of patient care services.[1] Community pharmacy is evolving towards offering a range of specialised services, such as asthma management and diabetes care.[2, 3] Before taking on new services it is, however, crucial that the profession review current core services to ensure that practice processes and procedures are designed to deliver quality pharmaceutical services. To achieve this goal community pharmacists should implement quality assurance and risk management processes.[4]

A model that is widely used as an approach for the assessment and evaluation of quality assurance in health care is one designed by Donabedian.[5] This approach assesses quality through the evaluation of three indicators, namely (1) the appraisal of structure; (2) the assessment of process; and (3) the assessment of outcome. The structure is defined as the physical or organisational properties; the process is defined as what is done; and the outcome as what is accomplished. These indicators overlap with the definition of risk management as defined in the Australian/New Zealand Standard on Risk Management, namely "the culture, processes and structures directed towards realising potential opportunities whilst managing adverse effects".[6] The Risk Management Standard provides a generic framework for establishing the context, identifying, analysing, evaluating, treating, monitoring and communicating risk.[7] Quality assurance activities and risk management techniques share the goal of minimising adverse patient outcomes. 
Donabedian's model and the Risk Management Standard could therefore be combined to formulate a pharmacy practice risk management framework, as summarised in Figure 1.

Insert Figure 1 here

Community pharmacy practice is influenced by the medicine regulatory framework. In Queensland the framework principally consists of the provisions set out in the Health (Drugs and Poisons) Regulation 1996 (the Regulation). Amendments were made to the Regulation during 2006 which introduced the need to have quality standards in place with regard to the dispensing and selling of scheduled medicines. Section 4 of the Regulation states that a quality standard refers to those standards recognised by the Pharmacists Board of Queensland. The Board, consistent with other Australian pharmacy regulatory authorities,[8] has endorsed all of the standards and guidelines developed by the main professional organisations, namely the Pharmaceutical Society of Australia (PSA) and the Society of Hospital Pharmacists of Australia. These standards and guidelines therefore have legislative backing and need to be implemented by pharmacists.[9] In accordance with section 376 of the Health Practitioners (Professional Standards) Act 1999 (Qld), the Board uses the standards as admissible evidence in disciplinary proceedings. The PSA standards specifically applicable to community pharmacy practice are the:[10] 
1. Professional practice standards, which consist of 17 individual standards, covering both core professional services such as dispensing and counselling, as well as selected speciality standards such as specialised drug information services; and

2. Standards for the provision of Pharmacist Only and Pharmacy Medicines in community pharmacy (S2/S3 standards).

An analysis by the Victorian Pharmacy Board that focussed on dispensing errors indicated that workload, distractions/interruptions and inadequacy of counselling all contributed towards dispensing errors.[11] Benrimoj's research focussed on the supply of Pharmacy Medicines (Schedule 2, sold only in pharmacies), and Pharmacist Only Medicines (Schedule 3, sold only by a pharmacist) and provided insight into the development of the S2/S3 Standards.[12] However, there is a dearth of information showing how Australian community pharmacists utilise and implement the practice standards.

Many of the functions performed by pharmacists involve both technical as well as cognitive components. For example, dispensing involves: (1) the clinical evaluation of the prescribed medicines, considering the patient's medical history and use of other medicines; (2) data entry and generation of the label; (3) selecting and labelling of the medicines; and (4) the provision of appropriate patient advice.[13, 14] Steps 1 and 4 require a pharmacist's professional expertise and judgement, whereas the other steps are mainly technical. The dispensing process is therefore a continuum of cognitive and 
technical functions which complicates workflow and the delegation of tasks in the dispensary.

Pharmacy support staff are increasingly being utilised to enable pharmacists to be released from technical functions and focus on patient care services. Standardisation of support staff qualifications is progressing through the National Community Pharmacy Training Package. As of March 2008, all Quality Care Pharmacy Program accredited pharmacies are required to ensure that staff handling Schedule 2 (S2) and Schedule 3 (S3) medicines have formal training.[15] However, grey areas regarding the roles of support staff remain.[8, 16]

A need was therefore identified to research the nature of community pharmacy practices in Australia, the roles of support staff and the ways in which pharmacists identify and manage risk in the context of the changing role of community pharmacy.[17, 18] This study explores the usefulness and implementation of practice standards in community pharmacy practice focusing on core services such as the dispensing of prescriptions and the supply of S2 and S3 medicines, staff utilisation and task delegation, and the recording of interventions.

\section{METHOD}


This study utilised a conceptual framework formulated through adopting Donabedian's approach and the Australian/New Zealand Standard on Risk Management to explore community pharmacy practices (Figure 1): the regulatory requirements (structure) were built into an interview guide; through interviews information was obtained about community pharmacy processes and procedures (process); that enabled the identification of pharmacists' perception of potential risks and the management thereof (outcomes). Ethical approval for the study was obtained from the Griffith University Human Research Ethics Committee.

\section{Semi-structured interviews}

A semi-structured interview guide was developed considering the aim of the project, literature findings,[11, 12, 14] peer opinion, an analysis of Queensland pharmacist disciplinary cases,[19] incidents reported to the Queensland branch of Pharmaceutical Defence Limited (PDL) (personal communication) and factual information such as practice standards and guidelines. Standards and guidelines specifically considered and integrated into the guide included:[13, 14, 20-22]

- The fundamental pharmacy practice professional practice standard;

- The dispensing professional practice standard;

- The guide to good dispensing;

- The counselling professional practice standard;

- The organisation of pharmacy practice professional practice standard; 
- Consumer medicine information and the pharmacist;

- Guidelines regarding generic substitution;

- The S2/S3 standards; and

- The Pharmacists Board of Queensland policy on the use of dispensary assistants.

The interview guide focused on the services, processes and procedures relating to the supply of over-the-counter (OTC) medicines, dispensing, incident management and recording and the integration of standards and guidelines.

The Gold Coast is a city with approximately 450,000 people located in South East Queensland and was selected for the study as being representative of urban and semi-rural Queensland. An expression of interest form with information sheet mailed to pharmacist managers of 95 Gold Coast pharmacies during July 2006 resulted in 13 expressions of interest being received, representing a broad range of pharmacist demographics. A further six pharmacists were approached directly for inclusion to further expand the demographic represenation. This process also ensured the inclusion of a pharmacy from a semi-rural area and a warehouse-type pharmacy. The process followed was therefore a combination of participant self-selection and purposeful selection.[23, 24] Recorded semi-structured interviews were conducted during August and September 2006 and field notes made. Although there were two more potential participants, the one relocated overseas and the other one continually deferred the interview. These two 
were not from the semi-rural or warehouse pharmacies and as no more subthemes were identified during the last few interviews the researcher was satisfied that saturation had been reached.

\section{Data analysis}

Some analytical techniques were borrowed from the grounded theory approach, whereby a theory is derived that is 'grounded' in the data and emphasises discovery with description and verification as secondary concerns.[23] Inductive analysis and constant comparison of categories and concepts assisted in clarifying the common and distinct themes emerging from the interviews. This is one of the conventions of interpretive research. As indicated by Patton, it 'depends on methods that take the researcher into and close to the real world so that the results and findings are grounded in the empirical world'.[24]

The interviews were transcribed verbatim, and analysed using NVivo 7 . The responses were grouped into several themes.

\section{RESULTS}

Two core pharmacy services that often require personal discretion and judgement will be discussed, namely dispensing and the supply of OTC 
medicines. This is followed by an analysis of staff and workforce issues and record keeping practices.

\section{Participant and pharmacy details}

Seventeen interviews (26 to 66 minutes in duration, average, 41 minutes) were conducted. Participants represented a wide range of demographics, experience and pharmacy types. (Table 1)

Insert Table 1 here

Community pharmacy practice experience predominated; one participant had extensive hospital pharmacy practice experience. All participants reported involvement with some form of continuous professional development.

\section{Provision of core pharmacy services}

Supply of OTC medicines

All of the interview participants expressed confidence in delegating the supply of OTC products to front shop pharmacy support staff:

"They all understand ..... the limits of what they can assist with. If the patient hasn't had the product before, if they have used the product and it's not working, if they are on other medications that may potentially 
interact with what they are requesting or if they have some sort of uncertainty about the condition or about the request they will then actually ask for the pharmacist and they are really quite happy to do that and we encourage that because they are very well trained and they are senior and they will often know the answer to the issue anyway because of their years in pharmacy but they still have a good understanding of when to refer to the pharmacist or request the pharmacist's advice." P10

All participants reported that staff used the PSA What-Stop-Go protocol[20] for providing S2 and S3 medicines as a tool for referring clients to the pharmacist. This protocol includes essential elements that should be covered to assist staff in gaining information regarding the appropriateness of the request and when to refer the client to a pharmacist. Five participants reported that staff also used other criteria and prompts to assist them in deciding when to refer customers to a pharmacist. However, the majority of participants did not have a well-defined system in place to monitor how well support staff used protocols. Instead, they said that their support staff referred patients on an ad hoc and unstructured basis: "Anything they are not sure of..." P11; "Well I guess the rule is if they are not sure about anything they have always got to ask." P13; and "If they get stuck with anything they refer them to me." P6

With regard to the sale of S3 medicines, all participants reported some pharmacist involvement which is in accordance with the Queensland requirements specified in the Health (Drugs and Poisons) Regulation 1996. 
However, the level of involvement varied considerably. For example, some pharmacies used forms with standard tick box questions to be completed by support staff; these were then given to the pharmacist to verify that the supply was therapeutically justified. In such instances pharmacists provided limited input regarding the appropriateness of the supply:

"Obviously they run it all past me but given that we are so busy I often don't have the time to go and individually see every S3 patient, so if I can see on their history that they have had it before which a lot of them do .......... it is a matter of OK, I have spoken to them before about it." P16

In general, the information gathering procedure and advice provided with regard to the supply of OTC medicines varied considerably between the pharmacies. The majority of participants did not regard the supply of OTC medicines as a pharmacist's direct role; they tended to mainly be involved when support staff identified that the supply required a pharmacist's intervention.

\section{Dispensing}

Considerable variation existed with regard to dispensing processes and the involvement of pharmacists in the provision of patient advice. Various of the participants made use of the PDL Guide to good dispensing[14] as a dispensing and training guide. This guide consists of the basic routine checks 
and procedures, built into eight steps to follow throughout dispensing. In contrast, the PSA Professional Practice Standard for dispensing[13] was neither known by the majority of participants nor used to develop dispensing processes and procedures. This endorsed standard comprises 13 criteria with indicators and additional notes; it is lengthy and does not identify which actions are absolutely essential to ensure patient safety as against those that are merely desirable.[18]

Dispensing workflow procedures were identified by a number of participants as specifically important in ensuring maximum efficiency and in meeting checking requirements:

"We have a protocol as well. We start from the left of the bench ......... We have a working regime, left to right. It comes through, label on the script then check both scripts. ${ }^{\star \star \star}$ will check them, then I will check them and put them in the box and then they get checked again either by myself, or ***. They basically get triple checked, but under no circumstances do the scripts sitting on that bench go out. That's our simple check." P7

The involvement of dispensary assistants in the dispensing process varied. Most participants did not clearly differentiate between pharmacist activities and dispensary assistant activities. Assistants seemed to undertake similar activities as pharmacists, including professional decision-making activities such as checking against the patient medication history. Roles appeared to 
be determined by the workload at a specific point in time, and the use of assistants did not necessarily release pharmacists to provide value-added patient care services:

"It really just depends on the demand. If it's busy then I am faster on the computers so I will actually do all of the dispensing and if I need to talk to the customer I communicate with coloured tags........ I guess the role is shared. Because we are only a small store the girls are capable of dispensing, they are capable of serving on the counter as am I, so it really just depends on how, what the demand is." P13

"I still do a lot of dispensing myself but if I am too busy they will do that." P11

No consistent workflow pattern was identified, and the delegated functions were often a reflection of a specific pharmacist's personal preference. Tools such as colour cards in the dispensed medication trays were frequently used to communicate information to support staff. For example, a colour card might identify a patient who required counselling by a pharmacist,or who needed specific information about recommended storage conditions. However, the degree of direct pharmacist contact with patients varied considerably amongst the participants, and the majority of participants stated that pharmacists counselled patients mainly when it was a new medication. The complexity of the prescribed medication and the availability of a pharmacist were also identified as important factors in the degree of 
pharmacist involvement in the provision of patient advice. Patients' prior knowledge and attitudes were used to determine the level of counselling to be provided, the member of staff who should provide the counselling, and the depth of information to be provided.

Participants reported frequent use of professional judgement in dispensing activities. Criteria used to supply Consumer Medicine Information (CMI) leaflets varied, for example one pharmacy had a protocol to ask all patients during the receipt of prescriptions whether they required written information, whereas the other pharmacies tended to only supply leaflets for newly prescribed medicines.

\section{Staff and workforce}

The majority of support staff either already had or were studying for a Certificate 2 or 3 in Community Pharmacy. In accordance with the Board Guidelines and policy regarding dispensary assistants,[22] all the participants indicated they only allowed support staff with or in training for a Certificate 3 qualification to assist in the dispensary.

Employment of pharmacy students offered certain advantages such as having 'support' staff with a solid academic background, motivating pharmacists to up-date their academic knowledge and providing access to electronic information resources not generally available in pharmacies: 
“... Micromedex. Students get it for free. It's a good product. It is very expensive but when the students are here we jump on. They have a logon." P3

Participants who employed pharmacist interns (pre-registration pharmacists) reported on the importance of providing a proper teaching environment:

"Because we constantly have a pre-reg here I would say we are pretty much spot on. They keep you on your toes." P8

“.... we always like to research anyway because we are in a teaching environment so the very fact that we have a pre-registration pharmacist, even if we know the answer, we would like to point out where we would have found it if we didn't know it." P17

\section{Record keeping}

Overall, pharmacist intervention record keeping was uncommon, especially detailed recording. Most did not keep any record of issuing CMI leaflets or other counselling tools, while only three stated they made electronic or other notes such as reminders to ask patients or carers follow-up questions on an irregular basis: 
"Whether it needs to be recorded? Where do you draw the line? Do you do it for all? That creates so much red tape. I think that comes down to personal discretion..." P7

None of the respondents kept records of 'near misses', i.e. errors identified before the medicine was supplied to the patient. Actual medication errors were recorded by participants only when they filed an incident report with their professional indemnity insurer (e.g. Guild Insurance), as those instances were considered of a more serious nature, and they feared potential litigation.

\section{DISCUSSION}

Donabedian's approach, namely that quality is associated with structure, process and outcome, combined with the Risk Management Standard as illustrated in Figure 1, provided a useful framework to evaluate existing services. This study investigated structure and process and identified that these elements were closely linked, as per Donabedian's theory, with outcomes. In this research the outcome of interest was not a patient outcome, but pharmacists' response to perceived or potential risks through their integration and utilisation of standards and guidelines into daily practices.

The interview analysis provided new insights and an understanding of contemporary pharmacy practice. Practice standards were not fully utilised 
and integrated into practice processes and procedures and may need to be reviewed with regard to usefulness and applicability. Areas to address to improve consistency of services for patients include the integration of prescribed standards, the procedures associated with dispensing and the supply of OTC medicines, the utilisation of pharmacy staff and record keeping

Pharmacy services are complex and processes are complicated by workload and staffing levels. This was demonstrated by the variation that existed in the services provided by the participants with regard to the supply of OTC medicines, with specific reference to the gathering of information and the provision of advice to clients. Pharmacists' involvement in the supply of OTC medicines focused mainly on the scheduling status of the products in order to comply with legislative requirements. This result is similar to previous findings.[25] Due to workload, a 'check box' approach had often been implemented whereby support staff would gather the information from the client and the pharmacist approved the provision of the medicine without personally talking to the client, unless the pharmacy's protocol triggered an intervention.

The supply of OTC therapies in some instances may or may not require a pharmacist's involvement and clinical judgement regardless of the schedule of the product. The delegation of the supply of OTC products to support staff therefore requires careful planning, training and workflow design so that a pharmacist is available to be involved when needed. 
Dispensary assistants' involvement in the dispensing of prescriptions varied, specifically with regard to workflow and the designation of tasks. Pharmacists were mainly involved with technical dispensing tasks and were therefore not continuously available to provide patient care services. Many of the patient contact functions were instead delegated to support staff. Releasing pharmacists to provide patient advice was influenced by the legislative requirement that all dispensed products must be personally checked and 'signed off' by a pharmacist prior to supply. There is therefore a need to redesign practice processes and the delegation of functions.

To comply with legislative and good pharmacy practice requirements, pharmacists are required to be available both in the dispensary and the front shop. This practical challenge was already identified by Strand in 1998 as limiting the provision of pharmaceutical care, as "Pharmacists could not dispense drugs and take care of patients at the same time." [26] Pharmacy managers therefore need to carefully design workflow models to release pharmacists to provide patient care services. However, the current financial model characterised by high costs and aggressive competition does not include remuneration for professional advice accompanying the supply of OTC medicines. Additionally, profit-margins on dispensed medicines do not adequately cover pharmacist advisory functions.

The recording of interventions, near misses and errors is critical as it provides a medical-legal record, facilitates the provision of quality and continuity of care, enables quality audits and peer review and allows the identification of 
system problems which should be addressed. Few participants kept such records, possibly reflecting unclear legislative requirements. While pharmacists are required to keep records of medication dispensed, the recording of interventions associated with the dispensing process is a grey area. Additionally, pharmacists may not appreciate the importance of record keeping in light of their changed role. The need to improve community pharmacy intervention record keeping has been identified previously.[27, 28]

Changes to dispensing software programs, training and remuneration of pharmacists can facilitate pharmacists focusing on how to adequately document, monitor and review the patient care they provide. [27, 29]

This study revealed variations in practice processes and procedures followed by community pharmacists with regard to core pharmacy services. Additionally, poor integration of practice standards suggests a need to review these for usefulness and practicability.

\section{Strengths and limitations of the study}

This research provided valuable information about community pharmacy practice and the integration of practice standards. The community pharmacists interviewed were mostly self-selected, willing to participate and be questioned. It is reasonable to assume that they were perhaps more motivated and committed to practice developments than those pharmacists who declined to be interviewed. The number of interview participants and the selection process limited the applicability of the findings. 


\section{CONCLUSION}

This study indicates that community pharmacy practice does not align well with prescribed practice standards. Practice processes and procedures need to be reviewed as well as prescribed practice standards to achieve better integration between these. Community pharmacy in Australia is at an exciting stage with more extended services being trialled through the fourth Community Pharmacy Agreement.[30] Workflow models and the delegation of tasks need to be re-evaluated considering these new roles and responsibilities to prevent a further divide between actual practices and required standards. The increased work demands on practice require of pharmacists to consider practice services carefully, prioritise and delegate functions to ensure that they are available to provide patient care services. As community pharmacy practice moves towards the provision of expanded primary health care services, community pharmacists need to utilise a quality cycle that will enable them to continuously plan, implement, review and improve existing and new practices.[31] 


\section{STRUCTURE Regulatory framework}

State and territory legislation applicable to pharmacy practice

legislation:

- Pharmacists Registration Act 2001

- Health Practitioner (Professional Standards) Act 1999

- Health Quality and Complaints Commissions Act 2006

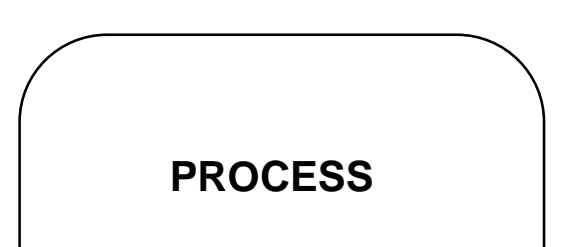

Community pharmacy practice:

- Processes \& procedures

- Utilisation of practice standards

Disciplinary action:

- Problem identification

\section{Delegated} legislation:

- Policies \& guidelines

- Code of Professional Conduct

- Professional Practice Standards

- Standards for the Provision of Pharmacist Only and Pharmacv

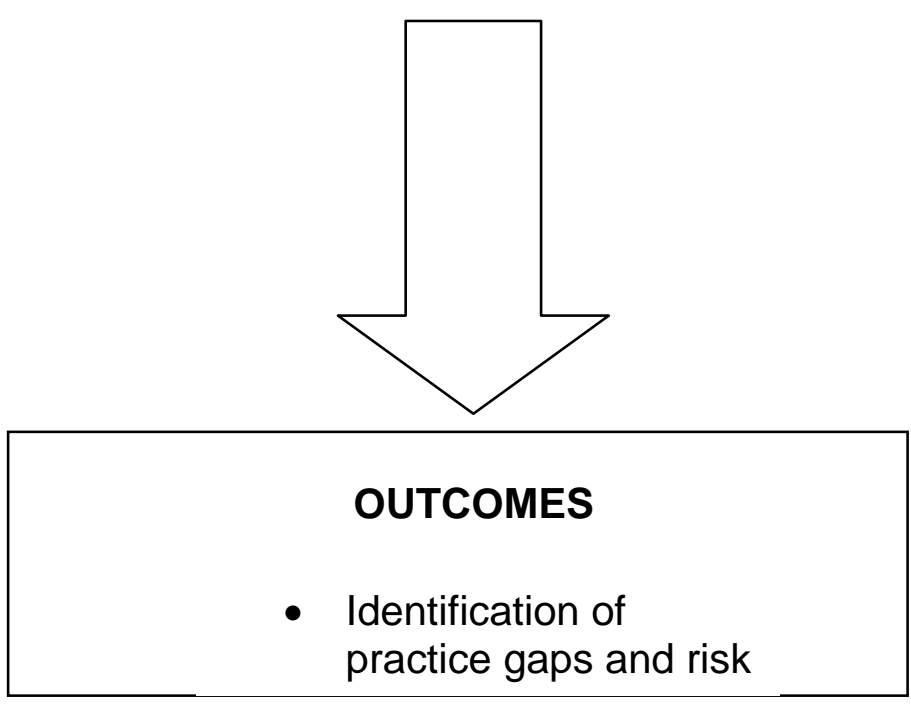

Figure 1: Risk management framework for community pharmacy practice. 
Table 1 Summary of interviewee characteristics

$\begin{array}{lcc}\text { Gender } & \text { No. } & (\%) \\ \text { Male } & 8 & (47.1) \\ \text { Female } & 9 & (52.9) \\ \text { Years of practice } & & \\ 1-5 & 4 & (23.5) \\ 6-10 & 2 & (11.8) \\ 11-20 & 5 & (29.4) \\ >20 & 6 & (35.3)\end{array}$

Position

Owner-manager $12 \quad$ (70.6)

Manager $\quad 5 \quad$ (29.4)

Professional affiliation/s (no.)

$\begin{array}{lll}0 & 3 & (17.6) \\ 1 & 6 & (35.3) \\ >1 & 8 & (47.1)\end{array}$

Pharmacy type

$\begin{array}{lcc}\text { Banner group }^{(\mathrm{a})} & 6 & (35.3) \\ \text { Independent } & 11 & (64.7)\end{array}$

Pharmacy location

Business street 6

Next to medical centre $\quad 5$

Shopping centre $\quad 5 \quad$ (29.4)

Private hospital $^{(b)} \quad 1$ 
a Banner group pharmacies are independently owned but share a common brand.

b This pharmacy served the hospital and community 
Acknowledgements:

The 17 community pharmacists who participated in the interviews.

\section{Conflict of interest:}

None

\section{Funding:}

The participant interviews were funded through a Queensland Pharmacy Research Trust grant. 


\section{REFERENCES}

[1] Benrimoj S, Frommer MS. Community pharmacy in Australia. Aust Health Rev 2004; 28: 238-46.

[2] Albrecht L, Roberts A, Benrimoj C, Williams K, Chen T, Asani P. Cognitive pharmaceutical services: financial facilitators. Aust Pharm 2006; 25 : 809-15.

[3] Berbatis CG, Sunderland VB, Joyce A, Bulsara M, Mills CR. Enhanced pharmacy services, barriers and facilitators in Australia' community pharmacies: Australia's National Pharmacy Database Project. Int J Pharm Pract 2007; 15: 185-91.

[4] Niselle P. Managing medical indemnity: must we choose between quality assurance and risk management? Med J Aust 2004; 181: 64-5.

[5] Donabedian A. Medical care appraisal - Quality and utilisation. Washington D.C.: American Public Health Association: 1969. p. 2

[6] Standards Association of Australia. Australian/New Zealand Standard Risk management: AS/NZS 4360, Homebush NSW: Standards Australia International Limited, 2004

[7] Standards Association of Australia. Australian/New Zealand Standard Handbook: Risk management guidelines: Companion to AS/NZS 4360, Homebush NSW: Standards Australia International Limited, 2004

[8] Hattingh HL, Smith NA, Searle J, King MA, Forrester K. Regulation of the pharmacy profession throughout Australia. J Pharm Pract Res 2007; 37: 174-7. 
[9] Pharmacists Board of Queensland. Bulletin No. 24. [document on the Internet]. PBQ; 2007. [cited 15 January 2007]. Available from http://www.pharmacyboard.qld.gov.au/publications.htm>.

[10] Pharmaceutical Society of Australia. Australian Pharmaceutical Formulary and Handbook. 20th ed. Canberra: Pharmaceutical Society of Australia: 2006. pp. 445-97

[11] Newgreen DB, Pressley JA, Marty SH. A survey of dispensing errors reported to the Pharmacy Board of Victoria, July 1998 to December 2004. Aust Pharm 2005; 24: 644 - 8.

[12] Benrimoj S. A Cost-Benefit Analysis of Pharmacist Only (S3) and Pharmacy Medicines (S2) and Risk-Based Evaluation of the Standards. [document on the Internet]. Aust Ins Health Ageing; 2006. [cited 3 June 2006]. Available from:

http://www.beta.guild.org.au/research/project_display.asp?id=246>.

[13] Pharmaceutical Society of Australia. Professional Practice Standards. 3rd ed. Curtin: Pharmaceutical Society of Australia; 2006. pp. 43-9

[14] Pharmaceutical Defence Limited (PDL). Guide to good dispensing. [document on the Internet]. PDL; 2005 [cited 12 December 2006]; Available from: http://www.pdl.org.au/mediacentre/dispensing_guide

[15] Pharmacy Guild of Australia. Quality Care Pharmacy Program (QCPP). [document on the Internet]. PGA; 2006 [cited 21 September 2006]; Available from: http://beta.guild.org.au/qcpp/

[16] Kinrade W. Workforce and career options for pharmacy assistants. [Report] Collingwood, Victoria: Healthcare Management Advisors, December 2004. 
[17] Berbatis CG, Sunderland VB, Joyce A, Bulsara M, Mills CR. National pharmacy database project [document on the Internet]. June 2003 [cited; 15 January 2008]. Available from: http://beta.guild.org.au/research/project_display.asp?id=239

[18] Hattingh L, Forrester K, Smith N, Searle J. Pharmacy practice developments: The potential impact on pharmacists' legal liability. J Law Med 2007; 14: 297-402.

[19] Hattingh HL, An investigation of community pharmacy risk management regulation and practices in the context of an expanding role [Dissertation]. Gold Coast, Australia: H.L.Hattingh, 2007

[20] Pharmaceutical Society of Australia. Standards for the Provision of Pharmacy Medicines and Pharmacist Only Medicines in Community Pharmacy. $2^{\text {nd }}$ ed. Curtin: Pharmaceutical Society of Australia; 2005

[21] Smith A, McLachlan A. Generic medicines: same difference? National Prescribing Service 2006; February: 1-4

[22] Pharmacists Board of Queensland. Policy: Dispensary assistants [document on the Internet]. Pharmacists Board of Queensland; 2006. [cited 15 January 2007]. Available from

http://www.pharmacyboard.qld.gov.au/Publications/Policies/Dispensary\%20A ssistants\%202005.pdf

[23] Merriam SB. Qualitative research in practice: Examples for discussion and analysis. San Francisco: Jossey-Bass 2002.

[24] Patton MQ. Qualitative research \& Evaluation methods. 3rd ed. London: Sage publications Ltd., 2002. ISBN 0-7619-1971-6, p. 125 
[25] Galbally R. Final report of the review of drugs, poisons and controlled substances legislation [Report]. Woden, ACT: Secretariat, Review of Drugs, Poisons and Controlled Substances Legislation, 2001.

[26] Strand L. Building a practice in pharmaceutical care. Pharm J 1998; 260: 874-6.

[27] Peterson GM, Liauw C, Elkerton J, McKenzie D, Fitzmaurice K. Development and evaluation of a computerised system for the provision and documentation of pharmacists' cognitive services [document on the Internet]; 2003 [cited 25 June 2007]. Available from: http://beta.guild.org.au/research/project_display.asp?id=269

[28] Gross Z. Benefits of recording interventions. Pharm J 2001; 267: 188.

[29] Westerlund TLO, Handl WHA, Marklund BRG, Allebeck P. Pharmacy Practitioners' Views on Computerized Documentation of Drug-Related Problems. Ann Pharmacother. 2003 ;37: 354-60.

[30] Pharmacy Guild of Australia. Professional pharmacy services. [document on the Internet]; 2008 [cited 15 March 2007] Available from: http://www.guild.org.au/pps/

[31] Australian Primary Care Collaborative. The improvement model. [document on the Internet]; 2007 [cited 200814 January]; Available from: http://www.apcc.org.au/ 